\title{
Einwirkung von Phthalsäureanhydrid auf einige Pyrrolderivate.
}

Von

Hans Fischer und Fr. Krollpfeiffer.

(Aus der II. Medizinischen Klinik zu München.)

(Der Redaktion zugegangen am 5. Oktober 1912.)

Die bis jetzt im Blut- und Gallenfarbstoff aufgefundenen Bausteine sind außer dem tetrasubstituierten Phyllopyrrol sämtlich trisubstituierte Pyrrole mit einer freien CH-Gruppe in $\alpha$-Stellung.

Zur Charakterisierung dieser Körper sind die Pikrate und die Azofarbstoffe mit Diazobenzolsulfosäure sehr geeignet, jedoch erschien es uns wünschenswert, weitere stabile Derivate kennen zu lernen. In dieser Absicht studierten wir die Einwirkung von Phthalsäureanhydrid auf substituierte Pyrrole mit einer in $\alpha$-Stellung befindlichen freien CH-Gruppe und erhielten in der Tat prachtvoll krystallisierende, sehr beständige Körper.

Die Einwirkung von Phthalsäureanhydrid auf Pyrrol selbst haben Ciamician und Dennstedt ${ }^{1}$ ) vor ca. 20 Jahren untersucht. Sie gelangten zu einer Verbindung, die wahrscheinlich folgende Konstitution besitzt.

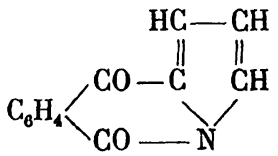

Der Körper zeigt die Eigenschaften eines Anhydrids. Er löst sich nicht in kalter Kalilauge, dagegen wird er in der Wärme durch dieses Reagens zu einer Säure aufgespalten, die als Pyrryl-phenylketon-carbonsäure anzusehen ist.<smiles>CCCCCC(=O)c1ccc[nH]1</smiles>

Die von uns gewonnenen Körper zeigen ein ganz analoges Verhalten und lassen sich gleichfalls durch Alkali in die zu-

1) Ber., Bd. 17, S. 2957. 
gehörigen, freien Säuren überführen. Es wurden die schön krystallisierenden Phthalide des Hämopyrrols, Kryptopyrrols und der Phonopyrrolcarbonsäure gewonnen. Auch Dimethylacetylpyrrol gibt eine schön krystallisierende Verbindung mit Phthalsäureanhydrid, ebenso Dimethylpyrrol. Während jedoch bei den zuerst erwähnten Verbindungen der Reaktionsmechanismus wahrscheinlich einfach durch Wasserabspaltung erfolgt, verlaufen beim Pyrrol und Dimethylpyrrol vielleicht kompliziertere Polymerisationsprozesse gleichzeitig, wenigstens geben die Derivate des Pyrrols und Dimethylpyrrols die Reaktion mit Dimethylamidobenzaldehyd nicht mehr. Allerdings gibt der Monoazofarbstoff des Dimethylpyrrols ${ }^{1}$ ) die Aldehydreaktion auch nicht.

Wie schon erwähnt, sind die Pyrrolenphthalide durch außerordentliche Beständigkeit und Krystallisationsfähigkeit ausgezeichnet, und scheinen daher besonders zur Isolierung von Pyrrolderivaten aus Gemischen geeignet. Als Beispiel führen wir die Gewinnung des Phthalids der Phonopyrrolcarbonsäure aus sirupöser Phonopyrrolcarbonsäure an. Diese Fraktion müßte hauptsächlich die von Piloty ${ }^{2}$ ) isolierte Xanthopyrrolcarbonsäure enthalten. Wir erhielten jedoch nicht das Phthalid dieser Säure, sondern das der Phonopyrrolcarbonsäure. Dieser Befund steht im Einklang mit den Resultaten der Untersuchung mittels Pikrinsäure; denn wir haben wiederholt aus dieser Fraktion Pikrate vom Schmelzpunkt $143^{\circ}$ isoliert, die Analysen aber ergaben immer nur auf Phonopyrrolcarbonsüure stimmende Werte. Für die Existenz der Xanthopyrrolcarbonsäure haben wir bis jetzt keinerlei Anhaltspunkte gewinnen können.

Von ganz besonderem Interesse ist die Einwirkung des Phthalsäureanhydrids auf Tetramethylpyrrol. Wider Erwarten wird hier glatt eine (wahrscheinlich $\alpha$-ständige) $\mathrm{CH}_{3}$-Gruppe abgesprengt, und es entsteht ein Trimethylpyrrolenphtalid. Die Bedeutung der Reaktion liegt auf der Hand. Wir hoffen, mit Hilfe dieser Reaktion durch Abspaltung des Phthalsäurerests einerseits zu den sonst schwer zugänglichen trialkylierten

1) Fischer und Bartholomäus, Diese Zeitschrift, Bd. 76, S. 479.

s) Piloty und Dormann, A., Bd. 388, S. 313 (1912). 
Pyrrolen mit einer freien $\alpha$-Stellung zu gelangen, anderseits besteht die Möglichkeit der Aufspaltung von Blut- und Gallenfarbstoffderivaten mit Hilfe des Phthalsäureanhydrids in der Vermutung, daß in den genannten Farbstoffen Pyrrolkerne in $\alpha$-Stellung durch eine $\mathrm{CH}_{2}$-Gruppe mit einander verknüpft sind.

2-4-Dimethyl-3-Acetyl-Pyrrolenphthalid.<smiles></smiles>

5,1 g 2-4-Dimethyl-3-Acetylpyrrol, 5,5 g Phthalsäureanhydrid und $8,2 \mathrm{ccm}$ Eisessig werden im zugeschmolzenen Rohr 5 Stunden auf $180-190^{\circ}$ erhitzt. Beim Erkalten ist der Röhreninhalt mit Krystallen durchsetzt. Die anhaftenden Schmieren werden durch Behandeln mit kaltem Alkohol nach dem Öffnen des Rohres leicht entfernt. Durch Umkrystallisieren aus viel heißem Alkohol erhält man den neuen Körper in schwach gelb gefärbten Nadeln vom Schmelzpunkt $183^{\circ}$. Ausbeute 4,5 g.

Zur Elementaranalyse wurde bei $100^{\circ}$ und $20 \mathrm{~mm}$ Druck über Phosphorpentoxyd getrocknet:

$0,3283 \mathrm{~g}$ Substanz gaben $0,1531 \mathrm{~g} \mathrm{H}_{2} \mathrm{O}$ und $0,8645 \mathrm{~g} \mathrm{CO}_{2}$. $0,3256 \mathrm{~g}$ Substanz gaben $15,6 \mathrm{ccm} \mathrm{N}$ bei $18^{\circ}$ und $716 \mathrm{~mm}$ $\mathrm{Hg}$ über $33 \%$ iger $\mathrm{KOH}$.

$$
\begin{array}{ll}
\mathrm{C}_{16} \mathrm{H}_{13} \mathrm{NO}_{3} & \text { Berechnet: } 71,88 \mathrm{C}, 4,90 \mathrm{H}, 5,25 \mathrm{~N} . \\
& \text { Gefunden: } 71,82 \mathrm{C}, 5,22 \mathrm{H}, 5,22 \mathrm{~N} .
\end{array}
$$

Der Körper ist in heißem Alkohol und Äther sehr schwer löslich, leichter in Eisessig. Die alkoholische Lösung reagiert neutral, die Reaktion mit Dimethylamidobenzaldehyd tritt erst nach dem Kochen mit Natronlauge auf.

Spaltung des Reaktionsproduktes mit 5\%iger Kalilauge.

0,5 g Dimethylacetylpyrrolenphthalid wurden mit $10 \mathrm{ccm}$ $5 \%$ iger Kalilauge bis zur Lösung gekocht. Beim Ansäuern 
Einwirkung von Phthalsäureanhydrid auf einige Pyrrolderivate. 269

der filtrierten Lösung entsteht eine voluminöse weiße Fällung. Aus Alkohol und viel Wasser erhält man den Körper in feinen farblosen Nädelchen. F. P. $176-178^{\circ}$. Ausbeute $0,4 \mathrm{~g} . \quad \mathbf{E r}$ löst sich in Natriumcarbonat unter Kohlensäurentwicklung.

\section{Kryptopyrrolphthalid.}

3,1 g Kryptopyrrol, 3,6 g Phthalsäureanhydrid und 5,5 ccm Eisessig werden 5 Stunden lang im zugeschmolzenen Rohr auf $180-190^{\circ}$ erhitzt. Nach dem Erkalten besteht der Röhreninhalt aus einer schwarzen Krystallmasse, verunreinigt durch ein dunkelbraunes Öl. Durch Absaugen und Waschen mit kaltem Alkohol erhält man braungelbe Nadeln, die nach mehrmaligem Umkrystallisieren aus heißem Alkohol eine zeisiggelbe Farbe annehmen. F. P. $169^{\circ}$. Ausbeute $1,5 \mathrm{~g}$. Die Reaktion mit Dimethylamidobenzaldehyd ist erst nach vorhergegangener Spaltung mit Alkali beim Kochen positiv. Zur Elementaranalyse wurde in der üblichen Weise bei $100^{\circ}$ getrocknet:

0,1166 g Substanz gaben 0,3222 g CO$_{2}$ und $0,0640 \mathrm{~g} \mathrm{H}_{2} \mathrm{O}$. $0,2275 \mathrm{~g}$ gaben $12,1 \mathrm{ccm} \mathrm{N}$ bei $20^{\circ}$ und $718 \mathrm{~mm}$ über $33 \%$ iger $\mathrm{KOH}$.

$\begin{array}{ll}\mathrm{C}_{16} \mathrm{H}_{15} \mathrm{NO}_{2} & \text { Berechnet: } 75,85 \mathrm{C}, 5,97 \mathrm{H}, 5,54 \mathrm{~N} \text {. } \\ & \text { Gefunden: } 75,43 \mathrm{C}, 6,15 \mathrm{H}, 5,76 \mathrm{~N} \text {. }\end{array}$

Die durch Aufspalten mittels Natronlauge und Fällen mit Salzsäure erhaltene freie Säure schmilzt nach Umkrystallisieren mit Alkohol bei $195^{\circ}$ unter Zersetzung.

\section{Phonopyrrolcarbonsäurephthalid.}

Das Ausgangsmaterial wurde durch Reduktion von Hämin gewonnen: 30 g Hämin lieferten 19,7 g Phonopyrrolcarbonsäurepikrat und 4,4 g sirupöses $\left.{ }^{1}\right)$ Phonopyrrolcarbonsäurengemisch.

1) Vergleiche Ber., Bd. 45, S. 1922 und 1985; alle bisherigen Befunde sprechen dafür, daß im Blutfarbstoff nicht die Xanthopyrrolcarbonsäure, sondern die von $\mathrm{H}$. Fis cher und $\mathrm{H}$. Röse im Bilirubin beobachtete isomere Phonopyrrolcarbonsäure vorkommt. Letztere ist übrigens, ebenso wie die Bilirubinsäure, auch von Piloty und Thannhauser (A., Bd. 390, S. 191 [1912]) durch Reduktion des Bilirubins mit Eisessig-Jodwasserstoff erhalten worden. Die beiden Herren haben das Resultat dieser schon in der I. Mitteilung über Gallenfarbstoff (Diese Zeitschrift, Bd. 73, S. 226 [1911]) von mir ausgeführten, erfolgversprechenden Untersuchungsmethode nicht abgewartet.

H. Fischer. 
Nach dem Umkrystallisieren des Phonopyrrolcarbonsäurepikrats wurde es mittels Salzsäure in gewohnter Weise in die schön krystallisierende Säure verwandelt.

6 g krystallisierte Phonopyrrolcarbonsäure, $6 \mathrm{~g}$ Phthalsäureanhydrid und $10 \mathrm{ccm}$ Eisessig werden 5 Stunden im Einschlußrohr auf $180-190^{\circ}$ erhitzt. Beim Erkalten ist der Röhreninhalt nahezu vollständig zu einem Krystallbrei erstarrt. Man saugt ab und wäscht mit Alkohol gut aus. Durch Umkrystallisieren aus viel heißem Alkohol erhält man schwach gelb gefärbte Nädelchen vom Schmelzpunkt 225-226 ${ }^{\circ}$. Ausbeute $5 \mathrm{~g}$ Rohprodukt. Auch hier tritt die Aldehydreaktion erst nach dem Aufspalten mit Alkali ein. Zur Elementaranalyse wurde wie üblich bei $100^{\circ}$ über Phosphorpentoxyd getrocknet :

0,2330 g Substanz gaben $0,5825 \mathrm{~g} \mathrm{CO}_{2}$ und $0,1150 \mathrm{~g} \mathrm{H}_{2} \mathrm{O}$.

$0,2770 \mathrm{~g}$ Substanz gaben $11,6 \mathrm{ccm} \mathrm{N}$ bei $15^{\circ}$ und $722 \mathrm{~mm}$ Druck über $33 \%$ iger $\mathrm{KOH}$.

$$
\begin{array}{ll}
\mathrm{C}_{17} \mathrm{H}_{15} \mathrm{NO}_{4} & \text { Berechnet: } 68,66 \mathrm{C}, 5,09 \mathrm{H}, 4,72 \mathrm{~N} \text {. } \\
& \text { Gefunden: } 68,18 \mathrm{C}, 5,52 \mathrm{H}, 4,66 \mathrm{~N} \text {. }
\end{array}
$$

Ein Versuch, aus dem Phthalid der Phonopyrrolcarbonsäure Kohlensäure abzuspalten durch Erhitzen auf 250-260', verlief negativ.

\section{Sirupöse Phonopyrrolcarbonsäure und Phthalsäureanhydrid.}

4,4 g sirupöse Phonopyrrolcarbonsäure, 4,4 Phthalsäureanhydrid und 6,6 ccm Eisessig wurden, wie bei der Phonopyrrolcarbonsäure beschrieben, erhitzt. Erhalten wurde $0,7 \mathrm{~g}$ krystallisiertes Material. Gelbe Nädelchen vom Schmelzpunkt $223^{\circ}$. Der Mischschmelzpunkt mit dem Phthalid der krystallisierten Phonopyrrolcarbonsäure wurde $\mathrm{zu} 224-225^{\circ}$ gefunden. Die Analyse bestätigte, daß das Phthalid der Phonopyrrolcarbonsäure vorlag.

$0,1622 \mathrm{~g}$ Substanz gaben $7,1 \mathrm{ccm} \mathrm{N}$ bei $16^{\circ}$ und $716 \mathrm{~mm}$ Druck über $33 \%$ iger $\mathrm{KOH}$.

Berechnet: $4,72 \mathrm{~N}$.

Gefunden: $4,82 \mathrm{~N}$. 
Einwirkung von Phthalsăureanhydrid auf einige Pyrrolderivate. 271

\section{Hämopyrrolphthalid.}

4,7 g Hämopyrrol, 5,5 g Phthalsäureanhydrid und 8,5 ccm Eisessig wurden, wie beim Kryptopyrrol beschrieben, verarbeitet. Die Ausbeute betrug $2,1 \mathrm{~g}$. Aus Alkohol erhält man derbe Prismen von zeisiggelber Farbe vom Schmelzpunkt $140^{\circ}$. Der Mischschmelzpunkt mit dem isomeren Kryptopyrrolphthalid wurde $\mathrm{zu} 116^{\circ}$ gefunden. Die Aldehydreaktion ist auch hier erst nach dem Kochen mit Natronlauge in der Hitze positiv.

Zur Elementaranalyse ${ }^{*}$ wurde in der üblichen Weise über Phosphorpentoxyd getrocknet:

$0,1655 \mathrm{~g}$ Substanz gaben $0,4583 \mathrm{~g} \mathrm{CO}_{2}$ und $0,0930 \mathrm{~g} \mathrm{H}_{2} \mathrm{O}$.

$0,2485 \mathrm{~g}$ Substanz gaben $12,7 \mathrm{ccm} \mathrm{N}$ bei $16^{\circ}$ und $722 \mathrm{~mm}$ $\mathrm{Hg}$ über $33 \%$ iger $\mathrm{KOH}$.

$$
\begin{array}{ll}
\mathrm{C}_{16} \mathrm{H}_{15} \mathrm{NO}_{2} & \text { Berechnet: } 75,85 \mathrm{C}, 5,97 \mathrm{H}, 5,54 \mathrm{~N} \text {. } \\
& \text { Gefunden: 75,52 C, 6,29 H, 5,67 N. }
\end{array}
$$

Tetramethylpyrrol und Phthalsäureanhydrid.

1,8 g Tetramethylpyrrol, 2,1 g Phthalsäureanhydrid und 3,1 ccm Eisessig wurden im Einschlußrohr 5 Stunden lang auf 180-190 ${ }^{\circ}$ erhitzt. Nach dem Erkalten war der Röhreninhalt von dunkelgefärbten Krystallen durchsetzt, die in der üblichen Weise gereinigt wurden. Aus Alkohol erhält man gelbe, derbe Prismen vom Schmelzpunkt $205^{\circ}$. Die A:dehydreaktion ist negativ, jedoch in der Hitze positiv nach der Aufspaltung mit Natronlauge. Die Analyse bestätigte, daß das Phthalid eines Trimethylpyrrols vorlag. Getrocknet wurde in der üblichen Weise :

$0,2435 \mathrm{~g}$ Substanz gaben $13,2 \mathrm{ccm} \mathrm{N}$ bei $18^{\circ}$ und $717 \mathrm{~mm}$ $\mathrm{Hg}$ über $33 \%$ iger $\mathrm{KOH}$.

Berechnet: $5,86 \mathrm{~N}$.

Gefunden: $5,94 \mathrm{~N}$.

\section{Aufspaltung mit Kalilauge.}

$2 \mathrm{~g}$ des Körpers werden in siedendem Alkohol gelöst und in die heiße Lösung ein Überschuß von $5 \%$ iger Kalilauge einlaufen gelassen. Nach zirka einstündigem Erhitzen auf dem 
272 Fischer u. Krollpfeiffer, Einwirkung von Phthalsäureanhydrid.

siedenden Wasserbad gewinnt man die freie Säure, die aus ca. 20\% igem Alkohol in derben Prismen krystallisiert. Ausbeute 1,7 g. Schmelzpunkt $204^{\circ}$ unter Zersetzung. Zur Analyse wurde wie üblich getrocknet:

$0,2394 \mathrm{~g}$ Substanz gaben $12,3 \mathrm{ccm} \mathrm{N}$ bei $19^{\circ}$ und $708 \mathrm{~mm}$ $\mathrm{Hg}$ über $33 \%$ iger $\mathrm{KOH}$.

Berechnet: $5,44 \mathrm{~N}$.

Gefunden : $5,52 \mathrm{~N}$.

Durch Einwirkung von Phthalsäureanhydrid auf 2-4Dimethylpyrrol unter den gleichen Bedingungen, wie auf die obigen Pyrrole, wurde in geringer Ausbeute ein bei ca. $170^{\circ}$ schmelzendes, krystallisierendes Produkt erhalten, das aber unter dem Mikroskop keinen einheitlichen Eindruck machte. Ebenso unbefriedigend verlief unter den geschilderten Bedingungen die Einwirkung des Phthalsäureanhydrids auf 2-4-5Trimethylpyrrol. Wir werden die Versuche fortsetzen. 